eCAM 2009; Page 129-131

doi:10.1093/ecam/nep046

\title{
Editorial
}

\section{eCAM: Integrative Genomics and Fecundity}

\section{Edwin L. Cooper}

Laboratory of Comparative Neuroimmunology, Department of Neurobiology, David Geffen School Of Medicine at UCLA, University of California, Los Angeles, CA 90095-1763, USA

Fecundity generally refers to the ability to reproduce. In biology and demography, fecundity is the potential reproductive capacity of an organism or population measured by the number of eggs or seeds. Fecundity under both genetic and environmental control is the major measure of fitness, is well studied in population ecology and can increase or decrease according to current conditions and certain regulating factors. In times of hardship for a population such as a dearth of food, the young or juveniles and eventually adult fecundity will decrease. eCAM fecundity refers to the ability of a scientific idea to open new lines of research or theoretical inquiry. If one quickly imagines, do any of these criteria really fit the current status of eCAM? I think so and will wet your appetite by giving some examples. First let us deal with eCAM and this analogy. Second we should identify a few eCAM contributions that are clearly in line with modern approaches to genomics and its overlays with eCAM.

eCAM fecundity concerns its continued growth. We have just received the encouraging news from Sophie Gilmour, Editorial Assistant, Oxford University Press. Here are the figures from her Executive Summary Q1 2009. There were 195 new manuscripts submitted from January to March 2009, compared to 71 during the same period in 2008. this represents a $175 \%$ increase. Because eCAM is evidence based, is it more poised to become a source of fertile analyses using combined approaches that are on the one hand CAM and on the other, first rate with respect to gene analyses that underlie disease? There lies, eCAM's beginning and possible future fecundity. Where are we with respect to understanding the genetic basis? It is desirable for eCAM to

For reprints and all correspondence: Edwin L. Cooper, Laboratory of Comparative Neuroimmunology, Department of Neurobiology, David Geffen School Of Medicine at UCLA, University of California,

Los Angeles, CA 90095-1763, USA. Tel: + 1-310-825-9567;

Fax: +1-310-825-2224; E-mail: cooper@mednet.ucla.edu continue in this direction with full speed. This should help in keeping the current level or even expand our current growth. Clearly eCAM has kept abreast of emerging technologies, as several examples will reveal.

A brief definition is appropriate. Integrated genomics analyses the genomes of organisms. In modern molecular biology an organism's genome is its hereditary information encoded in DNA. The field includes intensive efforts to determine the entire DNA sequence of organisms and to fine-scale genetic mapping efforts. Research of single genes does not include genomics unless the aim of this genetic pathway and functional information analysis is at consensus elucidating its effect on, place in, and response to the entire genome's networks. With this in mind, clearly CAM strategies should be devoted to zeroing in or pinpointing targets where effects might be operative and, with measured effects. Understanding the molecular mechanisms of disease requires an introduction of molecular diagnostics into medical practice (1). Current medicine employs only elements of molecular diagnostics, and usually on single genes. Medicine in the post-genomic era will utilize thousands of molecular markers associated with disease that are provided by high-throughput sequencing and functional genomic, proteomic and metabolomic studies.

This spectrum of techniques will link clinical medicine based on molecularly oriented diagnostics, predict and prevent disease. To this feat, large-scale and genomewide biological and medical data are essential and must be combined with biostatistical analyses and bioinformatic modeling of biological systems. Collecting, cataloging and comparison of data from molecular studies and subsequent development of conclusions create the fundamentals of systems biology (2). This highly complex analytical process reflects a new scientific paradigm and may be referred to as integrative genomics. Genomic studies provide us with methods to quickly analyze genes and 
their products en masse (3). Moving from the ancient to the modern, Ayurveda, the traditional Indian medicine (TIM) and traditional Chinese medicine (TCM) remain the most ancient yet living traditions. According to Patwardhan, et al., (2005) these are the two 'great traditions' backed by sound philosophical, experiential and experimental evidence (4). Recent advancements in the analytical and biological sciences, along with innovations in genomics and proteomics can play an important role in validation of these ancient therapies. Western scientific community views traditional medicines cautiously and emphasizes concerns related to research, development and quality. There is insistence on providing evidence.

Ventura (2005) relates this new paradigm to multiple holistic approaches: osteopathy, homeopathy, chiropractic, acupuncture, herbal and energy medicine and meditation (5). These interventions can modify the expression of different endorphin genes and those encoding for crucial transcription factors in cellular homeostasis. Extremely low frequency magnetic fields can prime the commitment to a myocardial lineage in mouse embryonic stem cells, suggesting that magnetic energy may direct stem cell differentiation into specific cellular phenotypes without employing gene transfer technologies. Different ginseng extracts modulate growth and differentiation in pluripotent cells and exert wound-healing and anti tumor effects through opposing activities on the vascular system so that ancient compounds could become targets for new approaches in cell therapy. Resulting CAM modalities may significantly affect signaling and transcriptional levels of cellular homeostasis. Thus functional genomics and proteomics and knowledge of cell signaling networks may contribute substantially to developing a molecular evidence-based CAM and pave the way to novel approaches in tissue engineering and regeneration. Molecular cartography is another term offered by Chiappelli (2005) (6). This refers to the multifaceted and intricate array of interacting genes and gene products that characterize functions and specialization of each individual cell and the context of cell-cell interaction, tissue and organ function, and system's biology.

Where have proteomics been innovative? 'Oketsu' is a pathophysiologic concept in Japanese traditional (Kampo) medicine, primarily denoting blood stasis/ stagnant syndrome. Matsumoto's team explored plasma protein biomarkers and/or diagnostic algorithms for 'Oketsu' (7). Sixteen rheumatoid arthritis (RA) patients were treated with keishibukuryogan (KBG), a representative Kampo medicine for improving 'Oketsu'. Hierarchical clustering gave three major clusters (I, II, III). Most $(68.4 \%)$ of 'Oketsu' samples were clustered in one cluster as the principal component of cluster I. The remaining 'Oketsu' profiles constituted a minor component of cluster II and were all derived from patients cured of the 'Oketsu' state at 12 weeks. Construction of a decision tree addressed the possibility of developing a diagnostic algorithm for 'Oketsu'. Results suggest that the pathophysiologic concept of Kampo medicine 'Oketsu' has a physical basis as measured by blood protein profiles. We may be able to establish objective criteria for diagnosing 'Oketsu' using a combination of proteomic and bioinformatics-based classification methods. Natural products are gaining increased applications in drug discovery and development so that there are emerging applications of DNA microarrays in pharmacodynamics, pharmacogenomics, toxicogenomics and quality control of herbal drugs and extracts (8).

Bacterial infections involving multi drug-resistant strains are one of ten leading causes of death and an important health problem in need of new antibacterial sources and agents (9). Four snake venoms (Agkistrodon rhodostoma, Bothrops jararaca, B. atrox and Lachesis muta) have been tested against 10 Gram-positive and Gram-negative drug-resistant clinical bacteria strains to identify them as new sources of potential antibacterial molecules. Mushrooms, similar to plants, have great potential for producing useful bioactive metabolites; they are a prolific resource for drugs (10). Responsible bioactive compounds belong to several chemical groups that are often polysaccharides or triterpenes. One species can possess bioactive compounds that can exert pharmacological effects. The best example is G. lucidum that contains $>120$ different triterpenes and polysaccharides, proteins and other bioactive compounds. The spectrum of detected pharmacological activities of mushrooms is broad. Dependent on increasing knowledge of chemistry, biotechnology and molecular biology of mushrooms as well as an improvement of screening methods (high throughput screening, genomics and proteomics), a rapid increase in the application of examples such as animal products and mushrooms for medicinal purposes can be expected.

Fecundity of eCAM is reflected in the increased number of manuscripts submitted recently and evidence that eCAM is poised to expand its repertoire to pinpoint targets of disease that could be modulated by CAM intervention using genomics. However in biomedical research and all other creative activity, no idea or movement is rarely clean cut and clear and free from intense scrutiny, even for an area so very pertinent and basic as genomics. Koenig (2009) points this out in a brief report under the section Biomedical Research. Genome Scans: Impatient for the Payoff (11). A debate in the genomics community about research strategy recently went public in commentaries published online by the New England Journal of Medicine (NEJM). A central disagreement concerns the likely value of chip-based genome scans to gauge inherited risks of developing common diseases. The discussion comes at a time when the U.S. National Human Research Institute (NHGRI) requests that scientists help direct its course in a new road map. Researchers contacted by Science agreed that far more work is 
required before physicians can properly utilize personal genomic assessments. Maybe eCAM will join the debate?

\section{References}

1. Ostrowski J. Integrative genomics-a basic and essential tool for the development of molecular medicine. Acta Pol Pharm. 2008;65:621-4.

2. Hood L, Heath JR, Phelps ME, Lin B. Systems biology and new technologies enable predictive and preventative medicine. Science. 2004;306:640-3.

3. Hocquette JF. Where are we in genomics? J Physiol Pharmacol. 2005;56(Suppl 3):37-70.

4. Patwardhan B, Warude D, Pushpangadan P, Bhatt N. Ayurveda and traditional Chinese medicine: A Comparative Overview. Evid Based Complement Altern Med 2005;2:465-73.

5. Ventura C. CAM and cell fate targeting: molecular and energetic insights into cell growth and differentiation. Evid Based Complement Altern Med 2005;2:277-83.

6. Chiappelli F. The molecular immunology of mucositis: implications for evidence-based research in alternative and complementary palliative treatments. Evid Based Complement Altern Med 2005;2:489-94

7. Matsumoto C, Kojima T, Ogawa K, Kamegai S, Oyama T, Shibagaki Y, Kawasaki T, Fujinaga H, Takahashi K, Hikiami H, Goto H, Kiga C, Koizumi K, Sakurai H, Muramoto H, Shimada Y, Yamamoto M, Terasawa K, Takeda S, Saik I. A proteomic approach for the diagnosis of 'Oketsu' (blood stasis), a pathophysiologic concept of Japanese traditional (kampo) medicine. Evid Based Complement Altern Med 2008;5:463-74.

8. Chavan P, Joshi K, Patwardhan B. DNA Microarrays in herbal drug research. Evid Based Complement Altern Med 2006;3:447-57.

9. Ferreira BL, Santos DO, Santos AL, Rodrigues CR, Freitas CC, Cabral LM, Castro HC. Comparative analysis of viperidae venoms antibacterial profile: a short communication for proteomics. Evid Based Complement Altern Med Advance (Access published on 2 September, 2008) doi:10.1093/ecam/nen052.

10. Lindequist U, Niedermeyer THJ, Jülich WD. The pharmacological potential of mushrooms. Evid Based Complement Altern Med 2005:2:285-99.

11. Koenig R. Genome scans: impatient for the payoff. Science 2009;324:448. 


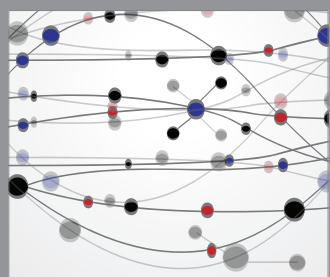

The Scientific World Journal
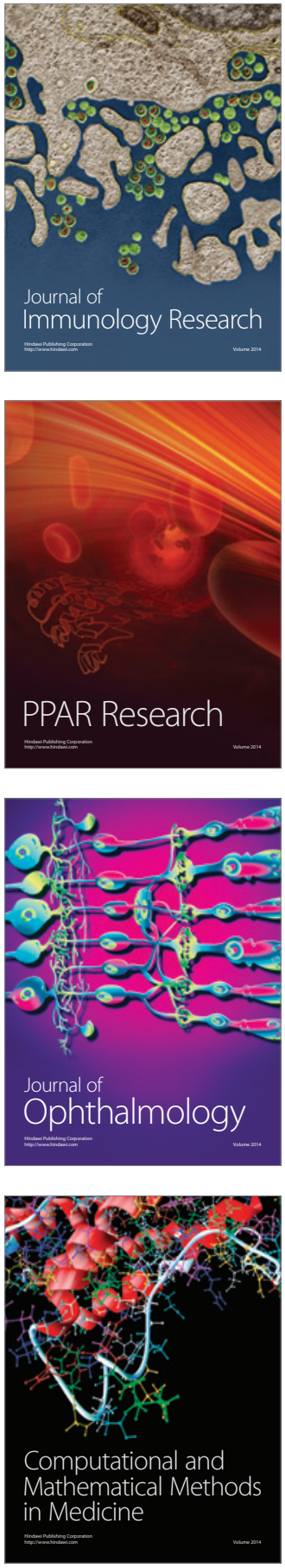

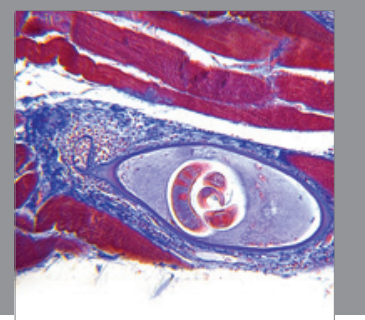

Gastroenterology

Research and Practice
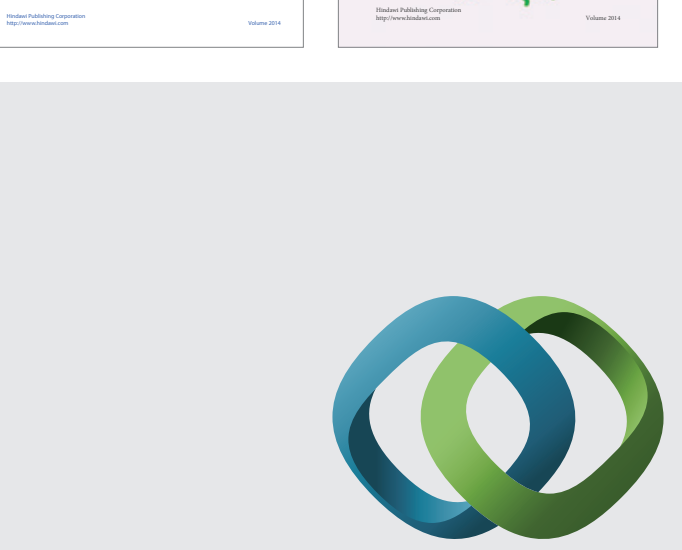

\section{Hindawi}

Submit your manuscripts at

http://www.hindawi.com
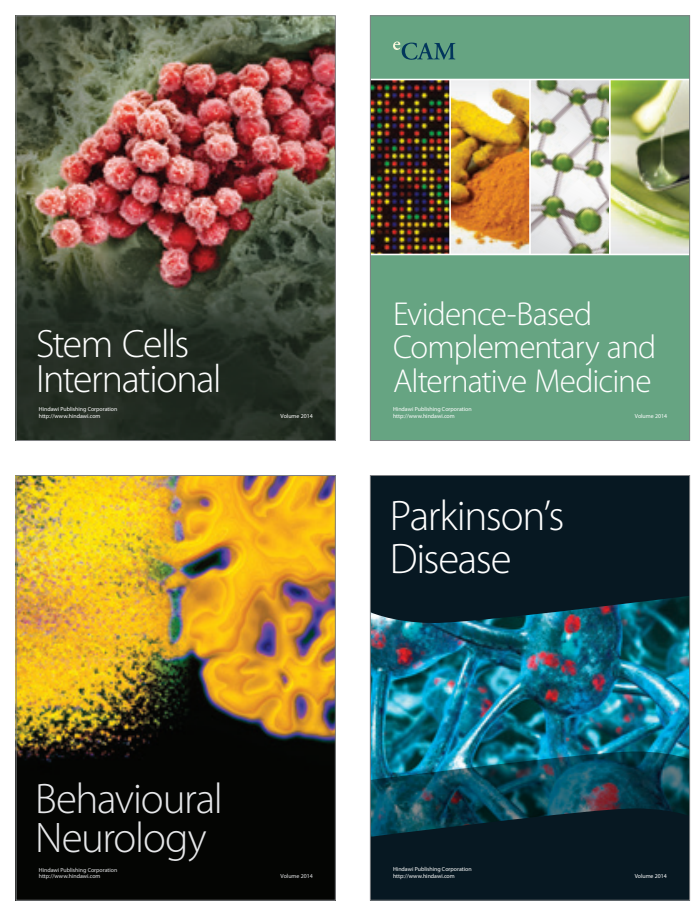

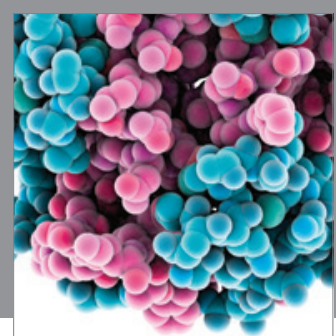

Journal of
Diabetes Research

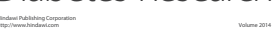

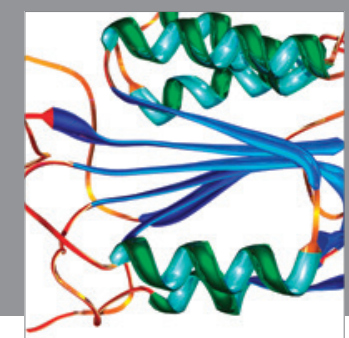

Disease Markers
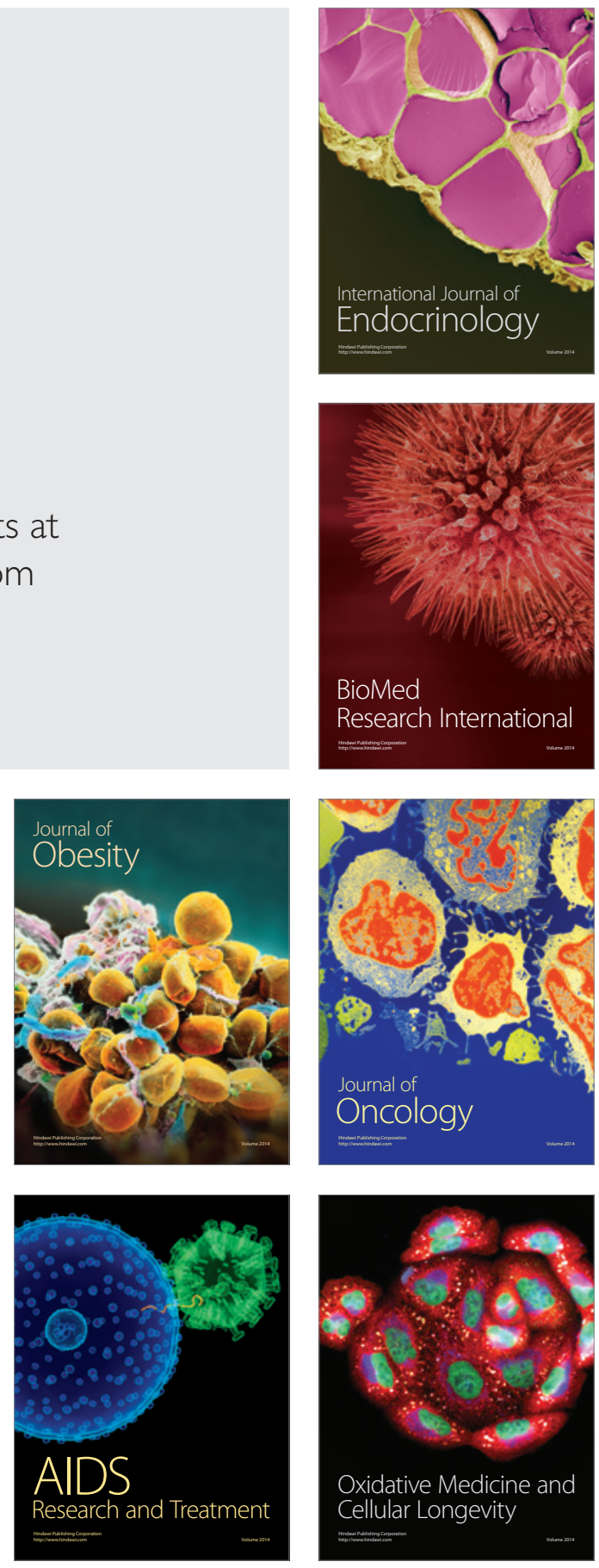